\title{
Wide-Area Delay-Dependent Adaptive Supervisory Control of Multi-machine Power System Based on Improve Free Weighting Matrix Approach*
}

\author{
Ziyong Zhang ${ }^{1}$, Zhijian Hu ${ }^{1}$, Yukai Liu ${ }^{1}$, Yang Gao ${ }^{2}$, He Wang ${ }^{1}$ Jianglei Suo ${ }^{1}$ \\ ${ }^{1}$ School of Electrical Engineering, Wuhan University, Wuhan, China. \\ ${ }^{2}$ Jiangsu Suzhou Power Supply Company, STATE GRID Corporation of China, Suzhou, China \\ Email: zzyhohai@163.com
}

Received January, 2013

\begin{abstract}
The paper demonstrates the possibility to enhance the damping of inter-area oscillations using Wide Area Measurement (WAM) based adaptive supervisory controller (ASC) which considers the wide-area signal transmission delays. The paper uses an LMI-based iterative nonlinear optimization algorithm to establish a method of designing state-feedback controllers for power systems with a time-varying delay. This method is based on the delay-dependent stabilization conditions obtained by the improved free weighting matrix (IFWM) approach. In the stabilization conditions, the upper bound of feedback signal's transmission delays is taken into consideration. Combining theories of state feedback control and state observer, the ASC is designed and time-delay output feedback robust controller is realized for power system. The ASC uses the input information from Phase Measurement Units (PMUs) in the system and dispatches supplementary control signals to the available local controllers. The design of the ASC is explained in detail and its performance validated by time domain simulations on a New England test power system (NETPS).
\end{abstract}

Keywords: Adaptive Supervisory Controller (ASC); Delay-dependent Damping Control; Power Oscillation; IFWM; LMI; Free Weighting Matrix Approach; Time-varying Delay; WAMS

\section{Introduction}

WITH the deregulation of power systems, many tie lines between control areas are driven to operate near their maximum capacity, especially those serving heavy load centers. Stressed operating conditions can increase the inter-area oscillation between different control areas and can even break up the system. The incidents of system outage resulting from these oscillations are of growing concern.

Over the past few decades, attention has been focused on designing controllers to dampen inter-area oscillations. The traditional method of damping inter-area oscillations is via the installation of power system stabilizers (PSS) which provide control action through the excitation control of generators[1]. Local PSSs are usually tuned based on several typical operating conditions of corresponding generators. z An inappropriate coordination among the local controllers may cause serious problems [2].

It has been suggested that centralized controllers using wide-area signals rely on the PMUs technology[3].

*This work is supported by Special Scientific and Research Funds for Doctoral Speciality of Institution of Higher Learning(20110141110032) and the Fundamental Research Funds for the Central Universities(2012207020205).
PMUs are used to capture the power system's dynamic data (e.g., voltages, currents, angles and frequency.) through synchronized measurements enabled by the GPS satellites. It has been shown that by using the remote signals the controller can enhance the damping of inter area oscillations and improve the overall dynamic performance of the power system[4]. A new PSS using two signals, the first to dampen the local mode in the area and the second, global signal, to dampen inter-area modes, is proposed in [5].

Application of techniques for designing robust power system damping controllers has been reported in the literature [6-9]. The solution to the control design problem based on the method of Riccati equations usually produces a controller that suffers from pole-zero cancellations between the system plant and the controller [10].

The application of the linear matrix inequality (LMI) approach as an alternative for damping controller design for PSS has been reported in [11,12]. A mixed-sensitivity based LMI approach has been applied to inter-area damping control design in [6,9]. In [9], FACTs are employed to damp inter-area oscillations. However, the cost of FACTs devices is quite high so that it currently re- 
stricts their wide use in power systems.

In the controller design, signal transmission delays should be considered $[7,8]$. The delays can typically be in the range of $0.3-1.0$ second[8]. As the delays are comparable to the time period of some of the critical inter-area modes, it should be accounted for in the design stage to ensure satisfactory control action.

In this paper, a wide-area adaptive supervisory controller (ASC) for robust stabilization of multi-machine power systems is proposed. Based on the IFWM approach and networked control system (NCS) theory [1316], the ASC is designed by delay-dependent stabilization condition. In the stabilization conditions, the upper bound of feedback signal's transmission delays is taken into consideration. The controller uses the input information (e.g. frequency, active power) provided by conveniently located PMUs and dispatches control signals to available local controllers. A particular feature of this controller is that it operates in addition to existing conventional PSSs and provides appropriate supplementary control signals only if and when needed. The performance and robustness of the controller are validated on a 4-generator 2-area test system.

\section{Strategy of Adaptive Supervisory Control}

A New England test power system (NETPS) is used as the example to analysis strategy of adaptive supervisory control. The model of the AVR with supplementary WAM signals is shown in Figure 1. In this figure, $V_{\mathrm{ASCi}}$ is the output signal of the ASC $[17,18]$ which is added to the AVR of each generator together with the output signal of four generators' local PSS. The structure of the ASC is shown in Figure 2.

\section{Controller Design Considering Signals Transmission Delay}

\subsection{Modeling of NCS-Based Power system with Network-Induced Delay}

Consider the following linear system:

$$
\dot{x}(t)=A x(t)+B u(t)
$$

where $x(t) \in R^{n}$ is the state vector; $u(t) \in R^{m}$ is the controlled input vector; and A and B are constant matrices with appropriate dimensions.

For convenience, we make the following assumptions [19].

Assumption 1 The NCS consists of a time-driven sensor, an event-driven controller, and an event-driven actuator, all of which are connected to a control network. The calculated delay is viewed as part of the network-induced delay between the controller and the actuator.

Assumption 2 The controller always uses the most recent data and discards old data. When old data arrive at the controller, they are treated as packed loss.

Assumption 3 The actual input obtained in (1) with a zero-order hold is piecewise constant function.

The control network itself induces transmission delays and dropped data that degrade the control performance of the NCSs-based power system. Based on these three assumptions, we can formulate a closed-loop power system with a memoryless state-feedback controller:

$$
\left\{\begin{array}{l}
\dot{x}(t)=A x(t)+B u(t) \\
u(t)=K x\left(t^{*}-\tau_{k}\right), t^{*} \in\left\{i_{k} h+\tau_{k}\right\}, k=1,2, \ldots,
\end{array}\right.
$$

where $h$ is the sampling period; $k=1,2,3, \ldots$ are the sequence numbers of the most recent data available to the controller, which are assumed not to change until new data arrive; $i_{k}$ is an integer denoting the sequence number of the sampling times of the sensor $\left\{i_{1}, i_{2}, i_{3}, \ldots\right\} \subseteq\{1,2,3, \ldots\}$; and $\tau_{k}$ is the delay from the instant $i_{k} h$, when a sensor node samples the sensor data from the plant, to the instant when the actuator transfers the data to the plant. Clearly,

$$
\bigcup_{k=1}^{\infty}\left[i_{k} h+\tau_{k}, i_{k+1} h+\tau_{k+1}\right)=\left[t_{0}, \infty\right) .
$$

From Assumption 2, $i_{k+1}>i_{k}$ is always true. The number of data packets lost or discarded is $i_{k+1}-i_{k}-1$. When $\left\{i_{1}, i_{2}, i_{3}, \ldots\right\}=\{1,2,3, \ldots\}$, no packets are dropped. If $i_{k+1}=i_{k}+1$, then $h+\tau_{k+1}>\tau_{k}$, which include

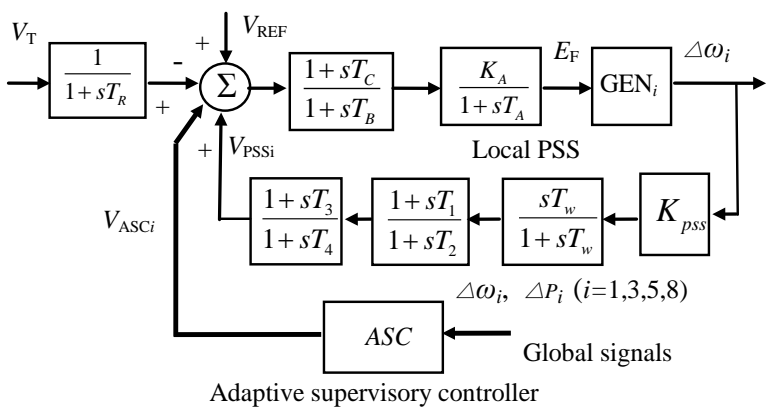

Figure 1. The designed model of $i^{\text {th }}$ exciter using the WAMs signals.

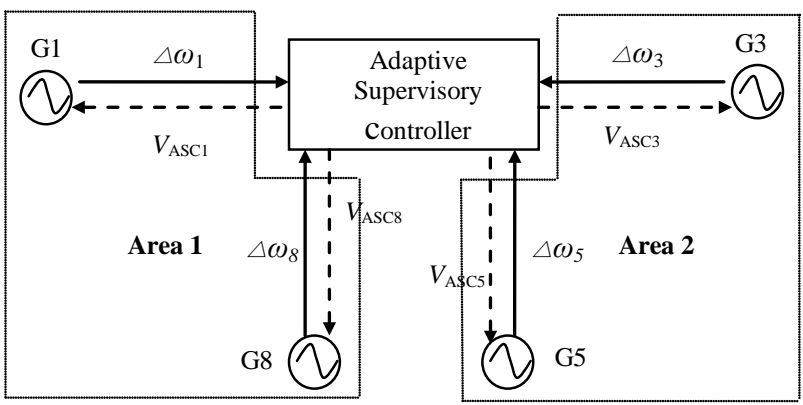

Figure 2. The input and output signals of the adaptive supervisory controller.

$\tau_{k}=\tau_{0}$ and $\tau_{k}<h$ as special cases. So, system (2) represents an NCS-based power system and takes the 
effects of both a network-induced delay and dropped data packets into account.

Below, we assume that $u(t)=0$ before the first control signal reaches the plant, and that a constant $\eta>0$ exists that

$$
\left(i_{k+1}-i_{k}\right) h+\tau_{k+1} \leq \eta, k=1,2, \ldots
$$

Based on this inequality, we can rewrite NCS (2) as

$$
\left\{\begin{aligned}
\dot{x}(t)= & A x(t)+B K x\left(i_{k} h\right), \quad t \in\left[i_{k} h+\tau_{k}, i_{k+1} h+\tau_{k+1}\right), \\
& k=1,2, \ldots, \\
x(t)= & x\left(t_{0}-\eta\right) e^{A\left(t-t_{0}+\eta\right)}=\phi(t),
\end{aligned}\right.
$$

where the initial condition function, $\phi(t)$, of the system is continuously differentiable and vector-valued.

\subsection{Delay-dependent Stability Analysis}

This section first present a new stability criterion for NCS (4), assuming the gain, $K$, is given.

Theorem 1. Consider NCS (4), given a scalar $\eta>0$, the system is asymptotically stable if there exist matrices $P>0, Q \geq 0, Z>0$, and $X=\left[\begin{array}{cc}X_{11} & X_{12} \\ * & X_{13}\end{array}\right] \geq 0$, and any appropriately dimensioned matrices $N=\left[\begin{array}{c}N_{1} \\ N_{2}\end{array}\right]$ and $M=\left[\begin{array}{ll}M_{1}^{T} & M_{2}^{T}\end{array}\right]^{T}$ such that the following matrix inequalities hold:

$$
\begin{gathered}
\phi=\left[\begin{array}{cccc}
\phi_{11} & \phi_{12} & -M_{1} & \eta A^{T} Z \\
* & \phi_{22} & -M_{2} & \eta K^{T} B^{T} Z \\
* & * & -Q & 0 \\
* & * & * & -\eta Z
\end{array}\right]<0, \\
\Psi_{1}=\left[\begin{array}{cc}
X & N \\
* & Z
\end{array}\right] \geq 0, \\
\Psi_{2}=\left[\begin{array}{cc}
X & M \\
* & Z
\end{array}\right] \geq 0,
\end{gathered}
$$

where

$$
\begin{aligned}
& \phi_{11}=P A+A^{T} P+Q+N_{1}+N_{1}^{T}+\eta X_{11}, \\
& \phi_{12}=P B K-N_{1}+N_{2}^{T}+M_{1}+\eta X_{12}, \\
& \phi_{13}=-N_{2}-N_{2}^{T}+M_{2}+M_{2}^{T}+\eta X_{22} .
\end{aligned}
$$

Proof. Choose the Lyapunov-Krasovskii functional candidate to be:

$$
\begin{aligned}
V\left(x_{t}\right)= & x^{T}(t) P x(t)+\int_{t-\eta}^{t} x^{T}(s) Q x(s) d s \\
& +\int_{-\eta}^{0} \int_{t+\theta}^{t} \dot{X}^{T}(s) Z \dot{x}(s) d s d \theta,
\end{aligned}
$$

where $P>0, Q \geq 0$, and $Z>0$ are to be determined.

From the Newton-Leibnitz formula, the following equations are true for any matrices $N=\left[\begin{array}{ll}N_{1}^{T} & N_{2}^{T}\end{array}\right]^{T}$ and $M=\left[\begin{array}{ll}M_{1}^{T} & M_{2}^{T}\end{array}\right]^{T}$ with appropriate dimensions:

$$
\begin{gathered}
0=2 \varsigma^{T}(t) N\left[x(t)-x\left(i_{k} h\right)-\int_{i_{k} h}^{t} \dot{x}(s) d s\right], \\
0=2 \varsigma^{T}(t) M\left[x\left(i_{k} h\right)-x(t-\eta)-\int_{t-\eta}^{i_{k} h} \dot{x}(s) d s\right],
\end{gathered}
$$

where $\varsigma(t)=\left[x^{T}(t), x^{T}\left(i_{k} h\right)\right]^{T}$. On the other hand, for any matrix $X=\left[\begin{array}{cc}X_{11} & X_{12} \\ * & X_{22}\end{array}\right] \geq 0$, the following equation holds:

$$
\begin{aligned}
0 & =\int_{t-\eta}^{t} \varsigma^{T}(t) X \varsigma(t) d s-\int_{t-\eta}^{t} \varsigma^{T}(t) X \varsigma(t) d s \\
& =\eta \varsigma^{T}(t) X \varsigma(t)-\int_{i_{k} h}^{t} \varsigma^{T}(t) X \varsigma(t) d s \int_{t-\eta}^{i_{k} h} \varsigma^{T}(t) X \varsigma(t) d s
\end{aligned}
$$

In addition, the following equation is also true $-\int_{t-\eta}^{t} \dot{x}^{T}(s) Z \dot{x}(s) d s=-\int_{i_{k} h}^{t} \dot{x}^{T}(s) Z \dot{x}(s) d s-\int_{t-\eta}^{i_{k} h} \dot{x}^{T}(s) Z \dot{x}(s) d s$

Calculating the derivative of $V\left(x_{t}\right)$ along the solutions of system (4) for $t \in\left[i_{k} h+\tau_{k}, i_{k+1} h+\tau_{k+1}\right)$, adding the right sides of (9)-(11) to it, and using (12) yield

$$
\begin{aligned}
\dot{V}\left(x_{t}\right)= & 2 x^{T}(t) P \dot{x}(t)+x^{T}(t) Q x(t)-x^{T}(t-\eta) Q x(t-\eta) \\
& +\eta \dot{x}^{T}(t) Z \dot{x}(t)-\int_{t-\eta}^{t} \dot{x}^{T}(t) Z \dot{x}(t) d s \\
= & 2 x^{T}(t) P \dot{x}(t)+x^{T}(t) Q x(t)-x^{T}(t-\eta) Q x(t-\eta) \\
& +\eta \dot{x}^{T}(t) Z \dot{x}(t)-\int_{i_{k} h}^{t} \dot{x}^{T}(s) Z \dot{x}(s) d s-\int_{t-\eta}^{i_{k} h} \dot{x}^{T}(s) Z \dot{x}(s) d s \\
& +2 \varsigma^{T}(t) N\left[x(t)-x\left(i_{k} h\right)-\int_{i_{k} h}^{t} \dot{x}(s) d s\right] \\
& +2 \varsigma^{T}(t) M\left[x\left(i_{k} h\right)-x(t-\eta)-\int_{t-\eta}^{i_{k} h} \dot{x}(s) d s\right] \\
& +\eta \varsigma^{T}(t) X \varsigma(t)-\int_{i_{k} h}^{t} \varsigma^{T}(t) X \varsigma(t) d s \int_{t-\eta}^{i_{k} h} \varsigma^{T}(t) X \varsigma(t) d: \\
= & \xi_{1}^{T}(t) \hat{\phi} \xi_{1}(t)-\int_{i_{k} h}^{t} \xi_{2}^{T}(t, s) \psi_{1} \xi_{2}(t, s) d s \\
- & \int_{t-\eta}^{i_{k} h} \xi_{2}^{T}(t, s) \psi_{2} \xi_{2}(t, s) d s
\end{aligned}
$$

where

$$
\begin{aligned}
& \hat{\phi}=\left[\begin{array}{ccc}
\phi_{11}+\eta A^{T} Z A & \phi_{12}+\eta A^{T} Z B K & -M_{1} \\
* & \phi_{22}+\eta K^{T} B^{T} Z B K & -M_{2} \\
* & * & -Q
\end{array}\right], \\
& \xi_{1}(t)=\left[\begin{array}{lll}
x^{T}(t), & x^{T}\left(i_{k} h\right), & x^{T}(t-\eta)
\end{array}\right]^{T}, \\
& \xi_{2}(t, s)=\left[\begin{array}{ll}
\varsigma^{T}(t), & \dot{x}^{T}(s)
\end{array}\right]^{T} .
\end{aligned}
$$

Thus, if $\psi_{i} \geq 0, i=1,2$, and $\hat{\phi}<0$, which is equivalent to (5) by the Schur complement, then $\dot{V}\left(x_{t}\right)<$ $-\varepsilon\|x(t)\|^{2}$ for a sufficiently small $\varepsilon>0$, which guarantees that system (4) is asymptotically stable. This com- 
pletes the proof.

When $M=0$ and $Q=\varepsilon I$ (where $\varepsilon>0$ is a sufficiently small scalar), the following corollary readily follows from Theorem 1.

Corollary 1 Consider NCS (4), given a scalar $\eta>0$, the system is asymptotically stable if there exist matrices $P>0, Z>0$, and $X=\left[\begin{array}{cc}X_{11} & X_{12} \\ * & X_{22}\end{array}\right] \geq 0$, and any appropriately dimensioned matrix $N=\left[\begin{array}{l}N_{1} \\ N_{2}\end{array}\right]$ such that matrix inequality (6) and the following one hold:

$$
\Xi=\left[\begin{array}{ccc}
\Xi_{11} & \Xi_{12} & \eta A^{T} Z \\
* & \Xi_{22} & \eta K^{T} B^{T} Z \\
* & * & -\eta Z
\end{array}\right]<0,
$$

where

$$
\begin{aligned}
& \Xi_{11}=P A+A^{T} P+N_{1}+N_{1}^{T}+\eta X_{11}, \\
& \Xi_{12}=P B K-N_{1}+N_{2}^{T}+\eta X_{12}, \\
& \Xi_{22}=-N_{2}-N_{2}^{T}+\eta X_{22} .
\end{aligned}
$$

\subsection{Design of State Feedback Controller}

Theorem 1 is extended to the design of a stabilization controller with gain $K$ for system (4).

Theorem 2. Consider NCS (4), for a given scalar $\eta>0$, if there exist matrices $L>0, W \geq 0, R>0$, and $Y=\left[\begin{array}{cc}Y_{11} & Y_{12} \\ * & Y_{22}\end{array}\right] \geq 0$, and any appropriately dimensioned matrices $S=\left[\begin{array}{ll}S_{1}^{T} & S_{2}^{T}\end{array}\right]^{T}, T=\left[\begin{array}{ll}T_{1}^{T} & T_{2}^{T}\end{array}\right]^{T}$, and $V$ such that the following matrix inequalities hold:

$$
\begin{gathered}
\Xi=\left[\begin{array}{cccc}
\Xi_{11} & \Xi_{12} & -T_{1} & \eta L A^{T} \\
* & \Xi_{22} & -T_{2} & \eta V^{T} B^{T} \\
* & * & -W & 0 \\
* & * & * & -\eta R
\end{array}\right]<0, \\
\Pi_{1}=\left[\begin{array}{cc}
Y & S \\
* & L R^{-1} L
\end{array}\right] \geq 0, \\
\Pi_{2}=\left[\begin{array}{cc}
Y & T \\
* & L R^{-1} L
\end{array}\right] \geq 0,
\end{gathered}
$$

where

$$
\begin{aligned}
& \Xi_{11}=A L+L A^{T}+W+S_{1}+S_{1}^{T}+\eta Y_{11}, \\
& \Xi_{12}=B V-S_{1}+S_{2}^{T}+T_{1}+\eta Y_{12}, \\
& \Xi_{22}=-S_{2}-S_{2}^{T}+T_{2}+T_{2}^{T}+\eta Y_{22} .
\end{aligned}
$$

then the system is asymptotically stable, and $K=V L^{-1}$ is a stabilizing controller gain.

Proof. Pre-and post-multiply $\phi$ in (5) by diag $\left\{P^{-1}, P^{-1}, P^{-1}, Z^{-1}\right\}$, and pre- and post-multiply $\psi_{i}, i=1,2$, in (6) and (7) by diag $\left\{P^{-1}, P^{-1}, P^{-1}\right\}$. Then, make the following changes to the variables:

$$
\begin{aligned}
& L=P^{-1}, R=Z^{-1}, V=K L, \\
& S_{i}=L N_{i} L, T_{i}=L M_{i} L, i=1,2, \\
& W=L Q L, Y=\operatorname{diag}\left\{P^{-1}, P^{-1}\right\} \cdot X \cdot \operatorname{diag}\left\{P^{-1}, P^{-1}\right\} .
\end{aligned}
$$

These manipulations yield matrix inequalities (15)(17). This completes the proof.

Note that the conditions in Theorem 2 are no longer LMI conditions due to the term $L R^{-1} L$ in (16) and (17). Thus, that cannot use a convex optimization algorithm to obtain an appropriate gain matrix, $K$, for the statefeedback controller. This problem can be solved by using the idea for solving a cone complementarity problem [20].

Define a new variable, $U$, for which $L R^{-1} L \geq U$; and let $P=L^{-1}, H=U^{-1}$, and $Z=R^{-1}$. Now, we convert the nonconvex problem into the following LMI-based nonlinear minimization problem:

Minimize $\operatorname{Tr}\{L P+U H+R Z\}$

Subject to (15) and

$$
\left\{\begin{array}{l}
{\left[\begin{array}{ll}
Y & S \\
* & U
\end{array}\right] \geq 0,\left[\begin{array}{ll}
Y & T \\
* & U
\end{array}\right] \geq 0,\left[\begin{array}{cc}
H & P \\
* & Z
\end{array}\right] \geq 0,} \\
{\left[\begin{array}{ll}
L & I \\
* & P
\end{array}\right] \geq 0,\left[\begin{array}{ll}
U & I \\
* & H
\end{array}\right] \geq 0,\left[\begin{array}{cc}
R & I \\
* & Z
\end{array}\right] \geq 0 .}
\end{array}\right.
$$

We use the ICCL algorithm to obtain $\eta_{\max }$ and $K_{\text {optimal }}$ for power systems because of its advantages.

\section{Algorithm.}

- Step1: Choose a sufficiently small initial $\eta>0$, such that there exists a feasible solution to (15) and (18). Set a specified number of iterations $N$.

- Step2:Find a feasible set of values satisfying (15) and (18), $\left(P_{0}, L_{0}, W, S, T, Y, Z_{0}, R_{0}, U_{0}, H_{0}, V\right)$.Set $k=0$

- Step3: Solve the following LMI problem for the variables $P, L, W, S, T, Y, Z, R, U, H, V$, and $K$ :

Minimize $\operatorname{Tr}\left\{L P_{k}+L_{k} P+U H_{k}+U_{k} H+R Z_{k}+R_{k} Z\right\}$

Subject to (15) and (18).

Set $P_{k+1}=P, L_{k+1}=L, U_{k+1}=U, H_{k+1}=H, R_{k+1}=R$, and $Z_{k+1}=Z$.

- Step4: For the $K$ obtained in step 3, if LMIs (15) and (18) are feasible for the variables $P, Q, Z, N, M$, and $X$, then set $\eta_{\max }=\eta$, increase $\eta$, and return to Step 2. If LMIs (5)-(7) are infeasible and without a specified number of iterations, then exit. Otherwise, set $k=k+1$ and go to Step 3 .

Figure 3 shows the flowchart of nonlinear iterative optimization algorithm for the state feedback controller design. This condition and nonlinear iterative optimization algorithm, which has an improved stop condition, are used to design a state-feedback networked controller. But the operating state variables of wide-area power sys- 
tem cannot be completely observed, it is necessary to use measurable states. Here, combining theories of state feedback control and state observer, the ASC is designed and time-delay output feedback robust control is realized for power system[21].

\section{Study System}

The New England test power system (NETPS) which consists of ten synchronous units in the system connected by weak tie-lines is shown in Figure 4[22]. This system is considered to be one of the benchmark models for performing studies on inter-area oscillations because of its realistic structure and availability of system parameters.

To validate the designed robust controller, the following disturbances were considered:

Case 1: A 2-phase fault at one of the lines between buses 6-11 followed by successful auto-reclosing of the circuit breaker after 4 cycles;

Case 2: A 3-phase fault at one of the lines between buses 6-11 followed by successful auto-reclosing of the circuit breaker after 4 cycles;

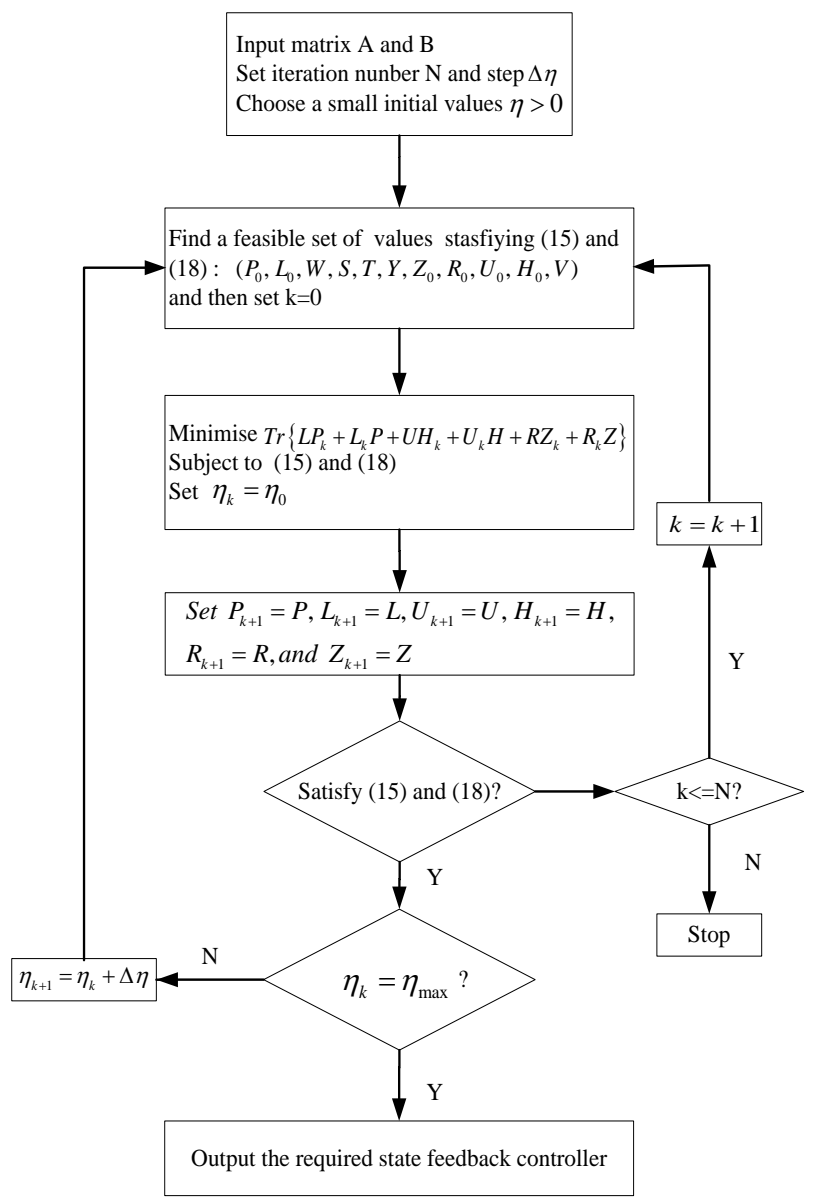

Figure 3. Flowchart of nonlinear iterative optimization algorithm.

\section{Simulation Results}

To validate the performance and robustness of the proposed control scheme involving ASC, simulations were carried out corresponding to the probable fault scenarios in the test system. If no time delay was considered, the simulation results were given in $[17,18]$. In each of the two cases, the total time delay for the feedback signals to arrive at the controller, then for the controller to send the signals to AVRs is 0.5 seconds.

\subsection{Case 1}

The rotor speed difference responses of multi-machine power system following a 2-phase fault are shown in Figures 5 and 6.

\subsection{Case 2}

The rotor speed difference responses of multi-machine power system following a 3-phase fault are shown in Figures 7 and 8.

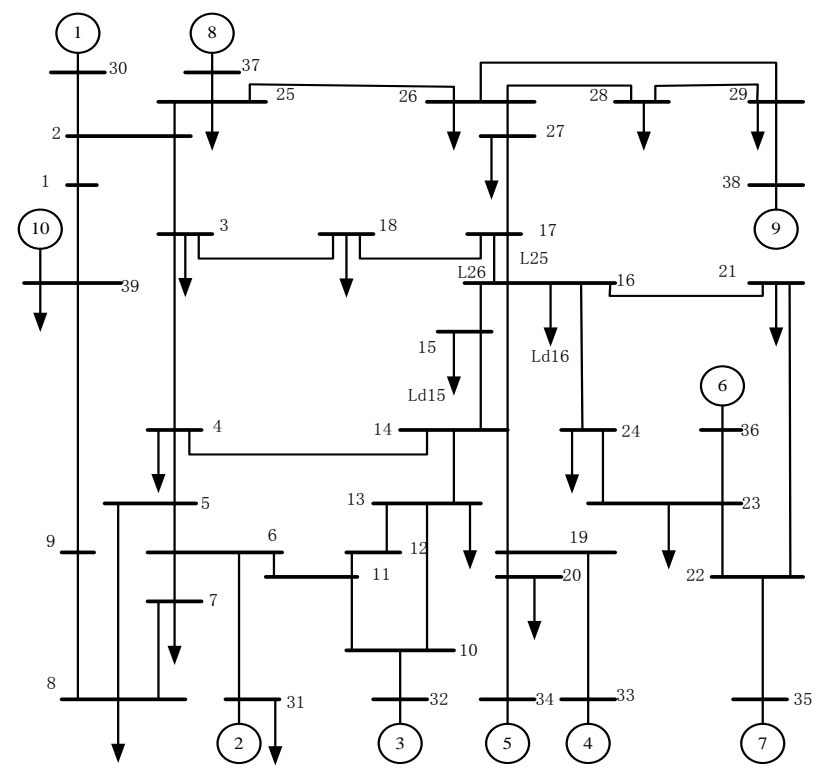

Figure 4. The New England test power system.

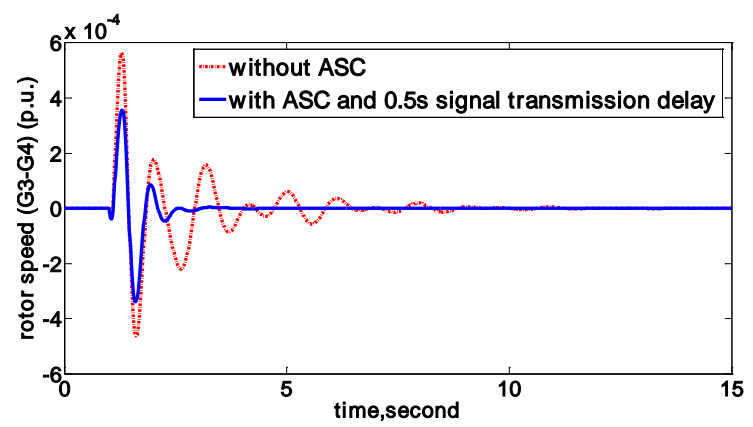

Figure 5. Rotor speed difference response of G3 and G4 following a 2-phase fault. 


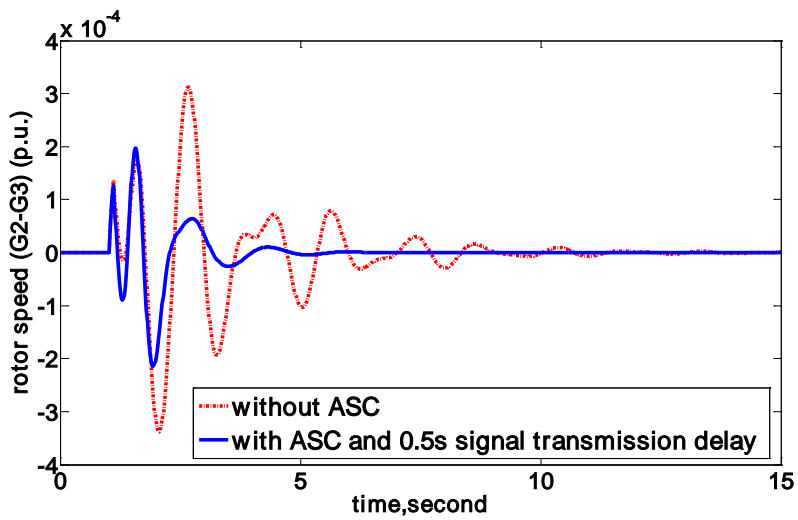

Figure 6. Rotor speed difference response of G2 and G3 following a 2-phase fault.

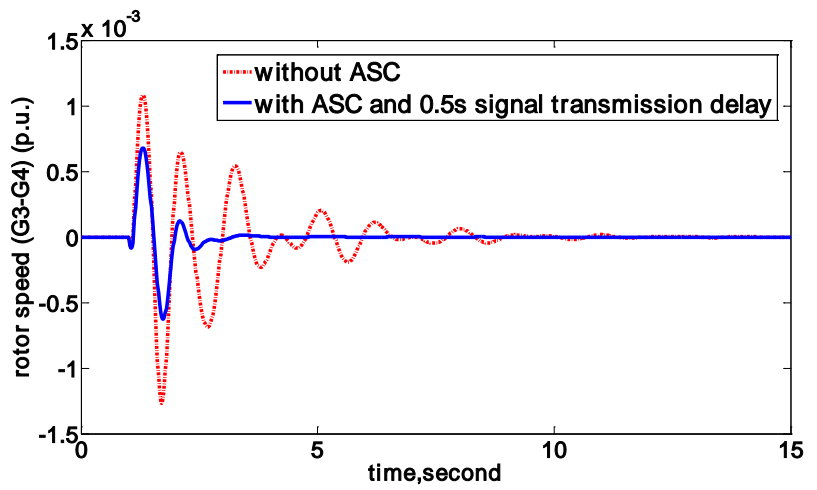

Figure 7. Rotor speed difference response of G3 and G4 following a 3-phase fault.

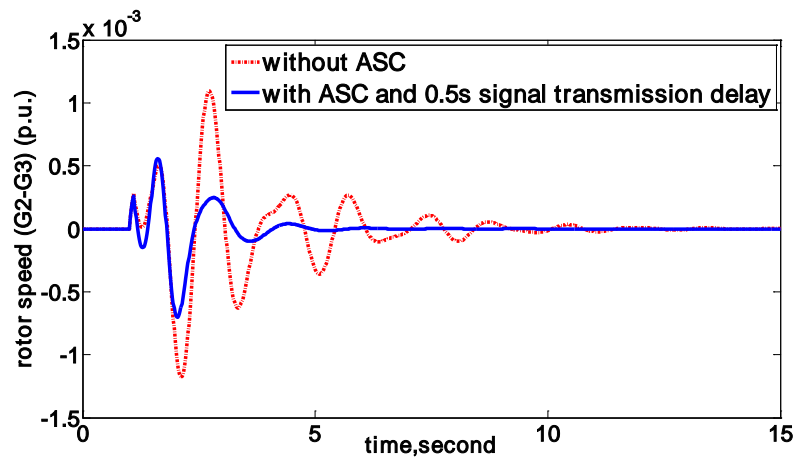

Figure 8. Rotor speed difference response of G2 and G3 following a 3-phase fault.

\section{Conclusions}

A wide-area adaptive supervisory controller (ASC) for the robust stabilization of multi-machine power systems accounting for the time delays of feedback signals from remote locations is proposed. This paper first uses the IFWM approach to establish an improved stability condition for NCSs-based power system that does not ignore any terms in the derivative of the Lyapunov-Krasovskii functional, but rather considers the relationships among a network-induced delay, its upper bound, and the difference between them. This condition and an ICCL algorithm, which has an improved stop condition, are used to design a state-feedback networked controller. Combining theories of state feedback control and state observer, the ASC is designed and time-delay output feedback robust control is realized for power system. With the control of the ASC, the system oscillations reduce considerably. Additionally, the ASC ensures system stability even in the case of cascading faults.

\section{REFERENCES}

[1] P. Kundur, Power system stability and control, New York: McGraw-Hill, 1994.

[2] G. J. Rogers, "The Application of Power System Stabilizers to a Multi-Generator Plant," IEEE Transacti Power Systems, Vol. 15, No. 1,2000. doi:10.1109/59.852143

[3] B. Bhargava, "Synchronized Phasor Measurement System Project at Southern California Edison Co.," in Proc. IEEE power Engineering Society Summer Meeting, 1999, pp. 16-22.

[4] H. Ni, G. T. Heydt and L. Mili, "Power System Stability Agents Using Robust Wide Area Control,” IEEE Transacti Power Systems, Vol. 17, No. 4, 2002, pp. 1123-1131.doi:10.1109/TPWRS.2002.805016

[5] M. E. Aboul-Ela, A. A. Sallam, J. D. McCalley and A. A. Fouad, "Damping Controller Design for Power System Oscillation,"IEEE Transacti Power Systems, Vol. 11, No. 2, , 1996, pp. 767-773. doi:10.1109/59.496152

[6] M. Klein, L. Le, G. Rogers, S. Farrokpay and N. Balu, "Hळ Damping Controller Design in Large Power System,” IEEE Transacti Power Systems, Vol. 10, No. 1, 1995, pp. 158-166. doi:10.1109/59.373938

[7] H. Wu, S. Tsakalis and G. T. Heydt, "Evaluation of Time Delay Effects to Wide-area Power System Stabilizer Design,” IEEE Transacti Power Systems, Vol. 19, No. 4, 2004, pp. 1935-1941. doi:10.1109/TPWRS.2004.836272

[8] B. Chaudhuri, R. Majumder and B. Pal, "Wide Area Measurement Based Stabilizing Control of Power System Considering Signal Transmission Delay,” IEEE Transacti Power Systems, Vol. 19, No. 4, 2004, pp. 1971-1979. doi:10.1109/TPWRS.2004.835669

[9] B. Chaudhuri, B. Pal, A. C. Zolotas, I. M. Jaimoukha and T. C. Green, "Mixed-sensitivity approach to Ho control of power system oscillations employing multiple facts devices,” IEEE Transacti Power Systems, Vol. 18, No. 3, 2003, pp. 1149-1156. doi:10.1109/TPWRS.2003.811311

[10] S. Skogestad and I. Postlethwaite, Multivariable feedback control, John Wiley and Sons, UK, 2001.

[11] P. Gahinet and P. Apkarian, “A Linear Matrix Inequality Approach to Ho Control," International Journal of Robust and Non-linear Control, Vol. 4, No. 4, 1994, pp. 421-448. doi:10.1002/rnc.4590040403

[12] C. Scherer, P. Gahinet and M. Chilali, "Multi-Objective 
Output Feedback Control Via LMI Optimization,” IEEE Transacti Automatic Control, Vol. 42, No. 7, 1997, pp. 896-911. doi:10.1109/9.599969

[13] Jianhua Z, Guolian H, Yunze W, et al., "Networked Control For Robotic Manipulators Via T-S Fuzzy Control And Descriptor Representation,” 2006.

[14] Zhigang X, Chaoyang D, Lijie X, et al., Networked Control of Guided Weapon Using Time-delay Switched System with Compensatory Model[C]. 2009.

[15] G. C. Goodwin, H. Haimovich, D. E. Quevedo, et al., “A Moving Horizon Approach to Networked Control System Design, Automatic Control, IEEE Transactions on, Vol. 49, No. 9, 2004, pp. 1427-1445. doi:10.1109/TAC.2004.834132

[16] G. P. Liu, Y. Q. Xia, D. Rees and W. S. Hu, "Design and Stability Criteria of Networked Predictive Control Systems With Random Network Delay in the Feedback Channel," Systems, Man, and Cybernetics, Part C: Applications and Reviews, IEEE Transactions on, Vol. 37, No. 2, 2007, pp. 173-184. doi:10.1109/TSMCC.2006.886987

[17] Z. Hu and J. V. Milanović, "Damping of Inter-area Oscillations by WAM Based Supplementary Controller," in Proc. IEEE PES GM 2007, 24-28 June 2007, Tampa,
Florida, USA.

[18] Z. Hu and J. V. Milanović, "The Effectiveness of WAM Based Adaptive Supervisory Controller for Global Stabilization of Power Systems," in Proc. IEEE Power Tech 2007, 1-5, July 2007, Lausanne, Switzerland.

[19] F.-L. Lian, J. K. Yook, D. M. Tilbury, et al., "Network Architecture and Communication Modules for Guaranteeing Acceptable Control and Communication Performance for Networked Multi-agent Systems,” Industrial Informatics, IEEE Transactions on, Vol. 2, No. 1, 2006, pp. 12-24. doi:10.1109/TII.2005.857611

[20] L. El Ghaoui, F. Oustry and M. Aitrami, “A Cone Complementarity Linearization Algorithm for Static Output-Feedback and Related Problems,” Automatic Control, IEEE Transactions on. Vol. 42, No. 8, 1997, pp. 1171-1176. doi:10.1109/9.618250

[21] F. Liu, R. Yokoyama, Y. Zhou, et al., SVC Robust Additional Damping Controller Design for Power System with Considering Time-delay of Wide-area Signals. 2010.

[22] E. Vittal, M. O'Malley and A. Keane, "Rotor Angle Stability With High Penetrations of Wind Generation," Power Systems, IEEE Transactions on, Vol. 27, No. 1, 2012, pp. 353-362. doi:10.1109/TPWRS.2011.2161097 